\title{
The Glory Program: Global Science from a Unique Spacecraft Integration
}

\author{
Jaya Bajpayee \\ NASA Goddard Space Flight Center \\ (301) 286-2688 \\ Jaya.bajpayee@gsfc.nasa.gov \\ Darcie Durham \\ Orbital Sciences Corporation \\ (703) 406-5836 \\ Durham.Darcie@Orbital.com \\ Thomas Itchkawich \\ Orbital Sciences Corporation \\ (703) $948-8538$ \\ Itchkawich.Tom@Orbital.com
}

\begin{abstract}
The Glory program is an Earth and Solar science mission designed to broaden science community knowledge of the environment. The causes and effects of global warming have become a concern in recent years and Glory aims to contribute to the knowledge base of the science community. Glory is designed for two functions: one is solar viewing to monitor the total solar irradiance and the other is observing the Earth's atmosphere for aerosol composition. The former is done with an active cavity radiometer, while the latter is accomplished with an aerosol polarimeter sensor to discern atmospheric particles.
\end{abstract}

The Glory program is managed by NASA Goddard Space Flight Center (GSFC) with Orbital Sciences in Dulles, VA as the prime contractor for the spacecraft bus, mission operations, and ground system. This paper will describe some of the more unique features of the Glory program including the integration and testing of the satellite and instruments as well as the science data processing. The spacecraft integration and test approach requires extensive analysis and additional planning to ensure existing components are successfully functioning with the new Glory components. The science mission data analysis requires development of mission unique processing systems and algorithms. Science data analysis and distribution will utilize our national assets at the Goddard Institute for Space Studies (GISS) and the University of Colorado's Laboratory for Atmospheric and Space Physics (LASP).
The satellite was originally designed and built for the Vegetation Canopy Lidar (VCL) mission, which was terminated in the middle of integration and testing due to payload development issues. The bus was then placed in secure storage in 2001 and removed from an environmentally controlled container in late 2003 to be refurbished to meet the Glory program requirements. Functional testing of all the components was done as a system at the start of the program, very different from a traditional program. The plan for Glory is to minimize any changes to the spacecraft in order to meet the Glory requirements. This means that the instrument designs must adhere to the existing interfaces and capabilities as much as possible.

Given Glory's unique history and the potential science return, the program is one of significant value to both the science community and the world. The findings Glory promises will improve our understanding of the drivers for global climate change for a minimal investment. The program hopes to show that reuse of existing government assets can result in a lower cost, and fully successful mission.

0-7803-9545-8/06/\$20.000 2006 IEEE

IEEEAC paper \#1414, Version 1 
TABLE OF CONTENTS

1. INTRODUCTION ....................................................... 2

2. HISTORY OF THE GLORY BUS ………………......... 2

3. MISSION OVERVIEW ............................................... 2

4. SCIENCE OVERVIEW .............................................. 3

5. SPACECRAFT INTEGRATION .................................. 5

6. SCIENCE DATA PROCESSING .................................... 7

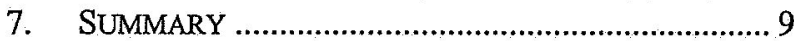

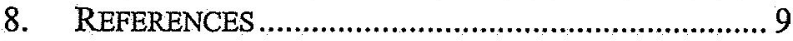

9. BIOGRAPHIES ...................................................... 9

\section{INTRODUCTION}

The Glory Mission is a dual science satellite incorporating both solar and Earth science on the observation platform. The nadir viewing Aerosol Polarimetry Sensor (APS) measures aerosols in the environment with the help of two cloud cameras to assist in differentiating between clouds and aerosols. On a solar viewing pointing platform of the satellite is a Total Irradiance Monitor to measure the longterm trends in solar activity. Because of the positioning of the satellite instruments as both solar and Earth viewing, the Glory phenomena* is the origin of the satellite's name.

The bus used for the Glory Mission has a unique history as it started out on another mission. The reuse of this bus and most of the components will be discussed with an emphasis on the integration and test approach. In addition, there will be a discussion of the mission science data (both solar and aerosol) and how this information will be processed and used.

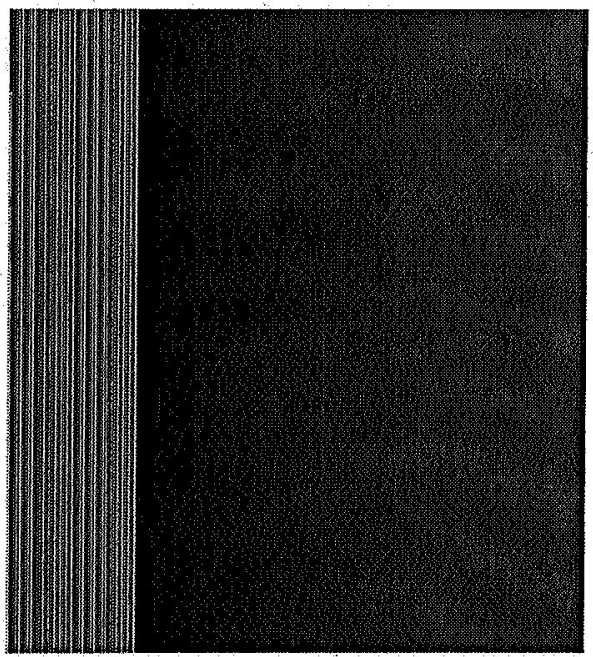

Figure 1 Glory Satellite

There are existing Earth and solar observing satellites, but none promise the science benefit and satellite manufacturing knowledge to be gained from this program.

\footnotetext{
- The Glory phenomena is the result of back-scattered light from individual water droplets that forms a diffraction (halo) pattern about the tip, of an object's shadow.
}

\section{HISTORY OF THE GLORY BUS}

The History of the Glory Bus is not your typical NASA scenario. Glory could easily have been called Phoenix, after the legendary bird that is resurrected from the ashes. The Glory bus began life as the bus for the GSFC Vegetation Canopy Lidar (VCL) mission. The bus contract for this mission was awarded to CTA Space Systems in 1997. The bus design and development began in earnest in 1998, just prior to CTA Space Systems purchase by Orbital Sciences. The bus heritage derives from the STEP series of spacecraft, flown for the Air Force on numerous missions (originally as Defense Systems, Inc or DSI).

The science payload for VCL was a suite of five lasers to measure the vegetation coverage around the world, especially in the tropical rainforests. Due to development problems with the lasers, the VCL mission was terminated. The VCL bus was put into controlled storage in 2001. In late 2002 the VCL bus was studied for use on the LASP AIM mission, followed by a directed study of flying the APS sensor with the Solar Irradiance Monitor (SIM) instrument that flew on SORCE. The positive results of this study were instrumental in the formation of the stand-alone Glory Mission, announced in July 2003 by the Administration as the. US contribution to the global Climate Change Research Initiative (CCRI).

In the VCL configuration, the bus architecture was redundant in most subsystems. The bus development had proceeded to the point where the bus was ready for system level testing and waiting for the science payload. The bus had been fully integrated, except for a few key components. The star tracker from the MAP mission was to be flown on VCL, but necessary changes had not been completed at the vendor. In addition the BlackJack GPS receiver from JPL had not been completed at JPL, and was never integrated to the bus. Finally, the flight card cage (C\&DH and ADACS) had not completed box-level acceptance testing, though it had been integrated with the bus

During the IEEE Aerospace conference last year (March 2005), the Glory mission was in dire straights due to budget shortfalls. However, Congress and NASA were able to work together to restore full funding for this critical science mission. So the Glory bus has had several instances of rising from the ashes. Fortunately for taxpayers and the global science community, the government investment in the bus will not be wasted.

\section{Mission OVERVIEW}

The Glory satellite is projected to launch from Vandenberg Air Force Base (VAFB) aboard a Taurus 2110. After the satellite has been placed into orbit, a 30-day on-orbit checkout will commence. Verification of initial insertion parameters will be made using the NASA Flight Dynamics Facility and the on-board GPS. Normal science operations 
will immediately follow a successful checkout of the bus and instruments and outgassing period.

The Glory satellite will be flown in a nominal $824 \mathrm{~km}$ sunsynchronous orbit with a 10:30am descending node. The Glory launch is scheduled for December of 2008 with a 3year mission lifetime and 5 year goal [1]. This orbit and mission altitude provide for the minimum duration required to observe seasonal and regional trends as well as characterize the evolution of aerosols during transient climate events.

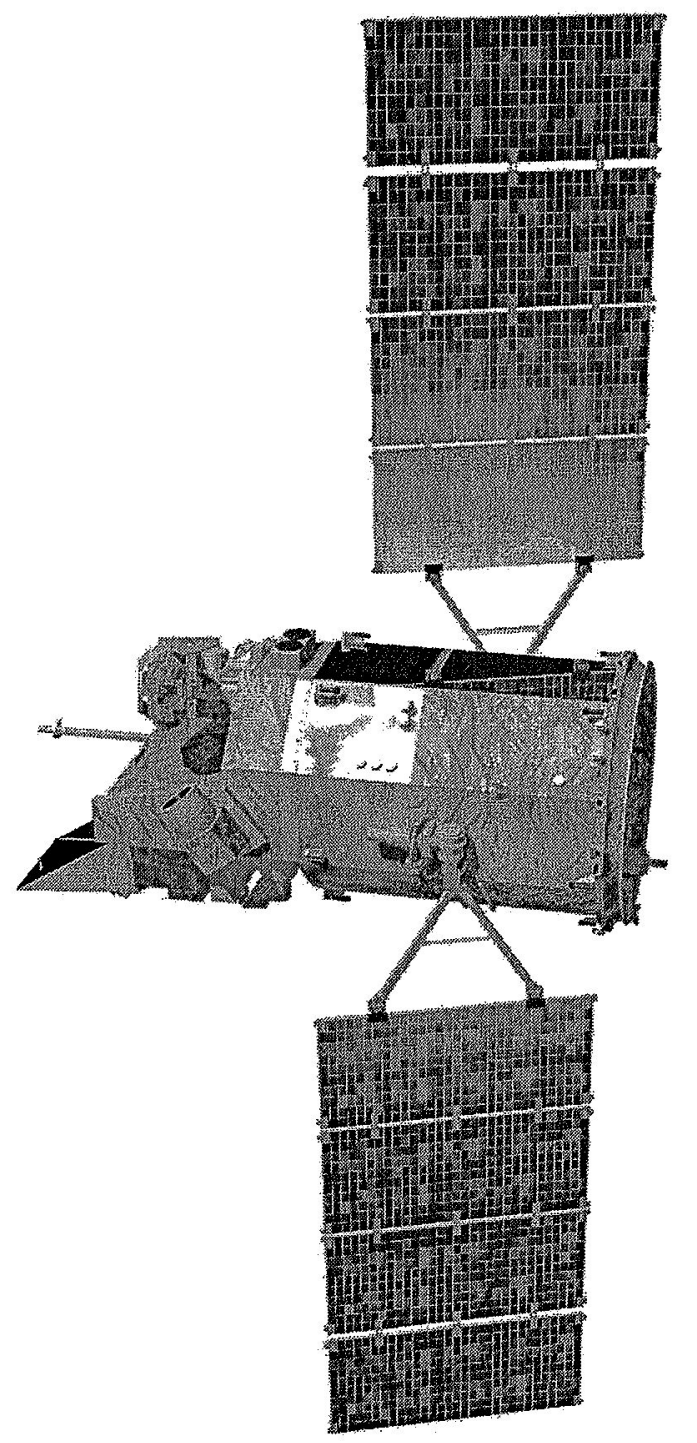

Figure 2 Satellite in Full Glory

Glory will be flown using Orbital's MAESTRO Command and Control software from the existing Mission Operations Center in Dulles, Virginia. Ground communications will use the Universal Space Network's. (USN) remote ground station in Poker Flat, Alaska as the primary station. USN sites in North Point, Hawaii and Perth, Australia will be used as backups for both the X-Band and S-Band passes. The TDRSS network will be used at the start of the misssion to ensure a safe launch and transition to early orbit checkout.

Glory will generate about 15 Gbits of data per day. All of this data will be downlinked during a single $X$-band pass via the USN network. Data will be routed to the GSFC/GISS and LASP Science Data Processing Centers, as well as the Orbital Mission Ops Center across. the IONET. Data is stored at the remote ground stations until data is confirmed to have been received at the SDPCs and MOC.

All of the Glory mission requirements can be met with the VCL bus, with the additional hardware mentioned above. Since Glory is flying at a much higher altitude, the ADACS can control to much tighter requirements than on VCL, although the Glory requirements are actually looser. Pointing accuracy and knowledge are easily met for Glory, as are the jitter and stability requirements. No science requirements had to be compromised to fit on the old VCL bus. This includes the data volume for both the APS and TIM instruments. The Cloud Camera data will be compressed so that 2 full days worth of data can be stored in the Solid State Recorder, which provides operational flexibility.

\section{SCIENCE OVERVIEW}

The Glory Mission will perform science measurements for aerosol and total solar irradiance using two instruments: Aerosol Polarimetry Sensor (APS) and Total Irradiance Monitor (TIM). The APS, in conjunction with two cloud cameras (CC), will measure natural and anthropogenic aerosols that play a crucial role in global climate and contribute to both warming and cooling of Earth's atmosphere[2].

There are multiple species of aerosols that affect the climate differently, specifically black carbon and sulfate aerosols. Black carbon aerosols can contribute to global warming by absorbing the Sun's radiation and re-radiating the Sun's energy as infrared radiation. This infrared energy is trapped by the Earth's atmosphere in much the same way that the windshield of an automobile contributes to a parked automobile heating up in the summer's sun. Sulfate aerosols, produced from the sulfur dioxide gas that spews out of a volcano or from the burning of sulfur-bearing fossil fuels, reflects the Sun's radiation out into space and typically cause cooling.

Aerosols, unlike greenhouse gases, have a short lifetime in the atmosphere (on the order of 1 to 2 weeks) [3]. Aerosols tend to mix with other agents and are transported up into the troposphere and then back down again. They are then transported by the winds across continents and disappear. Because aerosols can result from both natural and anthropogenic events, they are constantly replenished. 
Since the beginning of the industrial age, the anthropogenic aerosols have been increasing. Aerosols can also play a critical role in precipitation. Some species of aerosols may increase precipitation, while others may inhibit it. While the role of aerosols is recognized, because of the uncertainty of the composition of the aerosols in the atmosphere, there remains great uncertainty in the effect that atmospheric aerosols have on climate and weather.

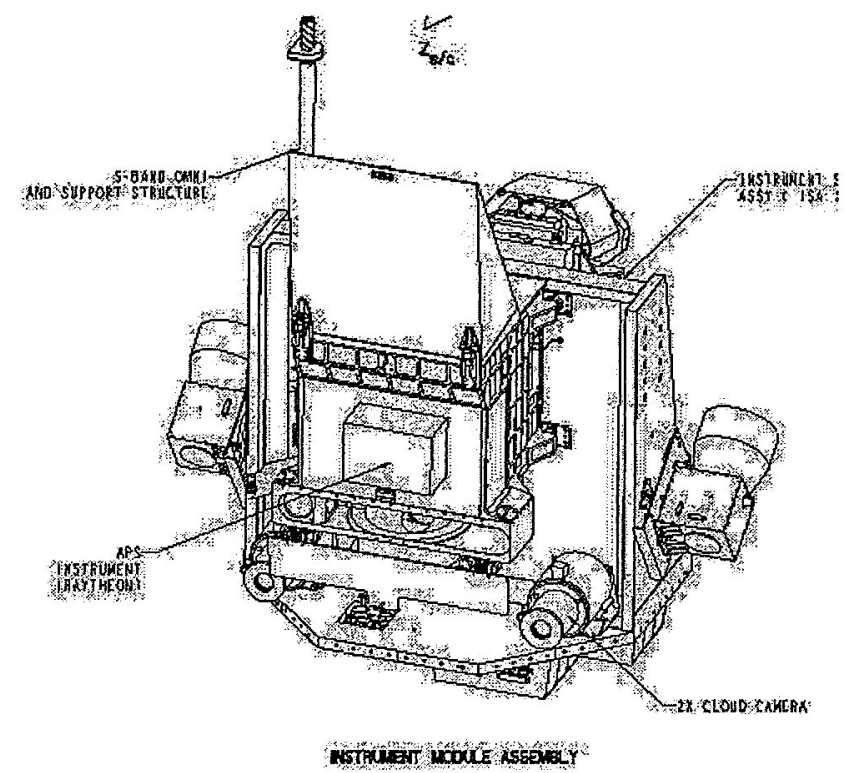

Figure 3 Glory Instrument Module Assembly

Total Solar Irradiance (TSI) measurements will be made by the TIM instrument. TSI together with the absorption and reflection of the radiation by the Earth's atmosphere determines the global average temperature of the Earth. The climate of the earth is directly affected by this balance between the intensity of the Sun and the response of the atmosphere. Changes in both the solar irradiance intensity and in the composition of the atmosphere can cause global climate change. Solar irradiance intensity is purely a natural phenomenon that fluctuates with the solar cycle, while the composition of the atmosphere is strongly influenced by the byproducts of modern industrial societies. Over the past century, the average temperature of the Earth has increased by about $0.5^{\circ} \mathrm{C}$ [4]. It is important to understand whether this change in global climate is a result of modern industry or if it is an effect of the natural solar phenomenon.

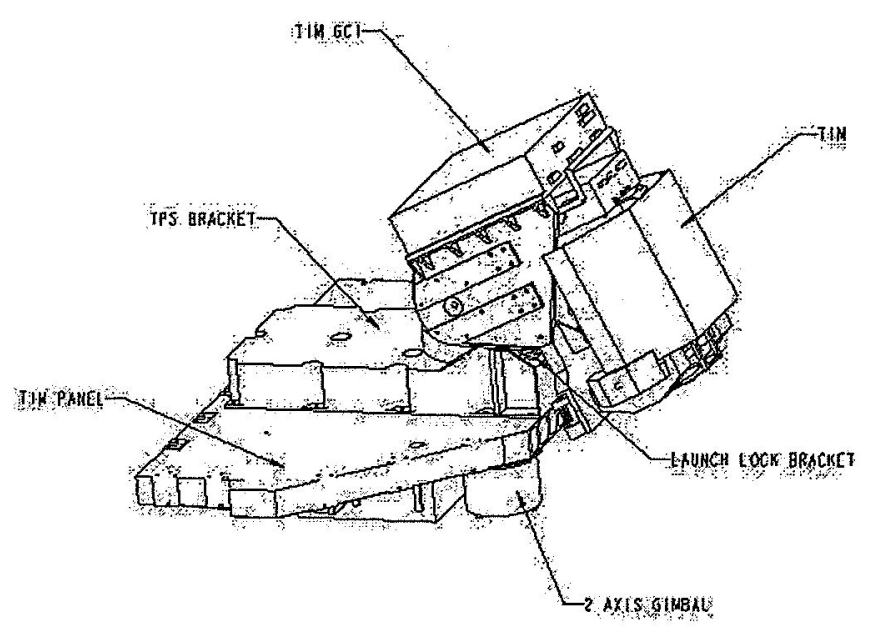

Figure 4 TIM Instrument

Understanding whether the increase in temperature and the concomitant climate change are byproducts of natural events or whether the changes are caused by anthropogenic sources is of primary importance to the establishment of scientifically and economically effective policy. The continued measurement of the TSI, at current state-of-art accuracy, without temporal gaps in the dataset, composes the solar irradiance requirement for the Glory Mission. It is essential that there be no temporal gaps in the data, as any measured shift in the atmosphere temperature must be correlated with the solar irradiance.

Glory's science goals, formulated to support the Climate Change Research Initiative, are as follows:

\section{Aerosol Research Science Goals}

Determination of the global distribution of natural and anthropogenic aerosols (black carbons, sulfates, etc.) and clouds.

- Determine the effect of aerosols on the earth's radiation including its natural and anthropogenic components.

- Determine the effects of aerosols on clouds and precipitation, including its natural and anthropogenic components.

- Investigate the potential for providing improved measurements of black carbons and dust absorption; this will provide more accurate estimates of their contribution to the climate forcing function.

\section{TSI Science Goals}

- Collect high accuracy measurements of total irradiance from the sun. 
- Continue TSI measurements for the long term climate record to determine the Sun's direct and indirect affects on Earth's climate.

\section{SPACECRAFT INTEGRATION}

The primary elements of the S/C Bus structure are the propulsion and core decks. Eight stringers connect the propulsion deck to the core deck and 8 shorter stringers attach to the core deck and extend upwards to support the Instrument Structure Assembly (ISA). Shear panels close out the eight sides of the structure.
The spacecraft bus components are mounted primarily on the core and propulsion plates. The propulsion plate is at the base of the cylinder and serves as the interface from the LV separation ring to the bus. The propulsion plate also houses the propulsion system, one of the 4 reaction wheels, and several other components. The majority of the Coarse Sun Sensors (CSS) are mounted in 8 sets of two around the circumference. The remaining bus components are mounted on both sides of the Core plate and the underside of the ISA.

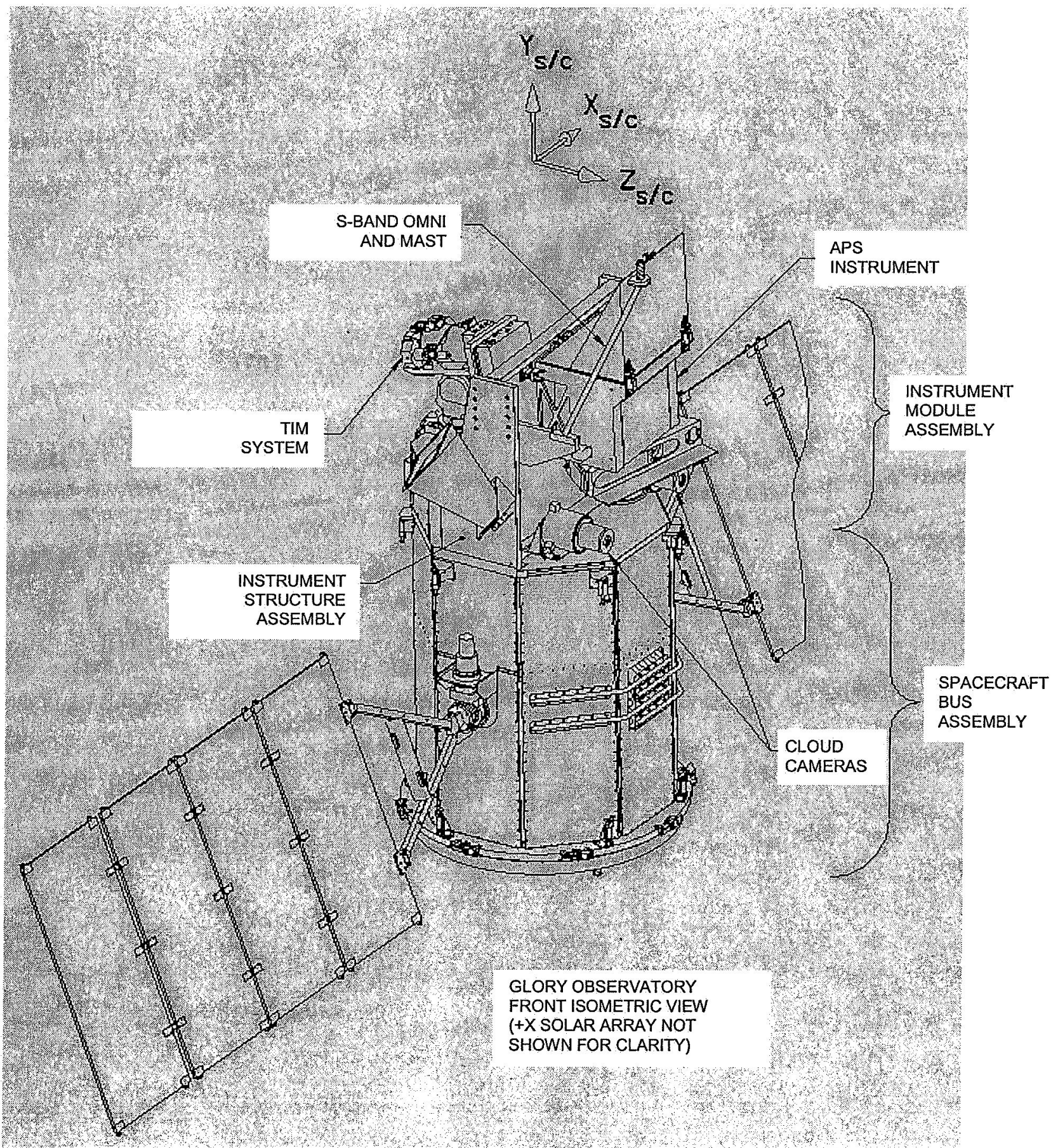

Figure 5 Detailed Glory Spacecraft 
The VCL layout has been left intact as much as possible to minimize changes to the wire harness. An added S-Band receiver is located in the space previously occupied by one of the two solar array drive assemblies (SADAs). Several bus components are mounted in the payload volume due to lack of deck space on the other two decks. These components are the fiber-optic gyro, the star trackers, the Payload Interface Processor (PIP), which is a new component for Glory, and the dual GPS receivers.

The state of the VCL bus when it was put into storage was not completely known due to loss of VCL personnel and incomplete documentation. It was decided that the best risk

posture was to de-integrate the bus electrically prior to proceeding with further testing. The components were physically left in the bus structure but all harness connectors were disconnected. This process facilitated two important things: understanding the state of the hardware integration and educating the Glory team on the VCL HW and its idiosyncrasies. This proved very valuable as it identified numerous issues with the harness and components as well as highlighted the differences between VCL and OV-3 implementations, of which many on the team were familiar.

The process integrated each component using the VCL component integration procedures. Each procedure was reviewed in detail and updated to ensure the safety of the flight HW, and to reflect Orbital's current ISO processes. In some cases, the procedures were replaced with the more rigorously tested OV-3 procedures, then modified for Glory particulars/differences. One item of note during the integration occurred when the Dual Card Cage (DCC) was integrated. When VCL was shut down, the non-flight EDU unit was in the bus. It was decided to integrate with this unit, since the flight dual card cage (DCC) had not been fully acceptance tested after rework on several cards. As the unit was integrated, it was discovered that the EDU was not fully populated, contrary to documentation indicating that it was. The DCC was missing all RF cards, limiting testing of the RF HW and system, other than in terms of RF power. It was decided to complete testing that could be done with this unit while in parallel completing the component audit process for the flight DCC. This was done, no issues were found that jeopardized the safety of the flight HW, and the flight unit was integrated, allowing completion of integration of the remaining components.

The culmination of the integration effort was a comprehensive Evaluation Performance Test (EPT). This EPT was a subset of the Comprehensive Performance Test (CPT) that tested all of the HW functions of the components but not the mission level software aspects. The reasoning behind this approach was to determine actions necessary to assure fully functional HW, not test software that would be replaced with a new baseline. The testing was invasive enough to test all features of the hardware and ensure that the HW-SW integration had no issues. The EPT was successful in uncovering several issues that required further investigation. In addition, the audit process also uncovered a few issues that the integrated bus was employed to troubleshoot. ISO processes were followed to implement testing. All troubleshooting steps and procedures were reviewed and approved by the MRB in order to ensure that the safety of the flight HW was paramount. Any problems that were determined to be SW only, were documented in the system and deferred to testing of the new SW baseline.

Finally, as part of the re-integration and EPT process, the new ground software was introduced to the system. VCL had used an expensive COTS product that did not make sense to buy on Glory again. Instead, Orbital is using our proven MAESTRO ground software. This software is used for component testing, satellite integration and test, and finally for mission operations. The initial Glory command and telemetry database was effectively built up from scratch, since the old data was not in a useful, available form from the previous ground system contractor. MAESTRO has been used in this manner on numerous Orbital programs with great success.

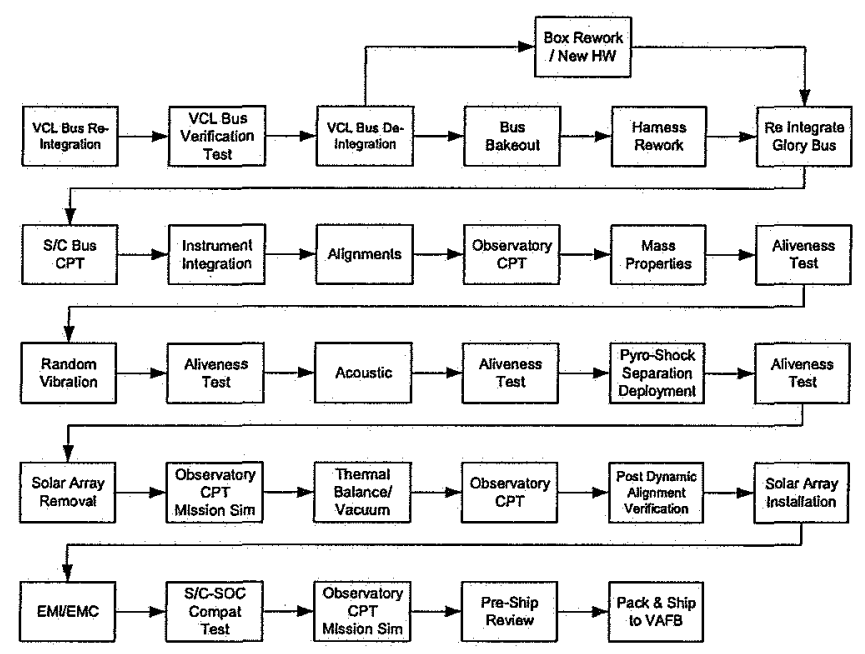

Figüre 6 Glory I\&T Flow

The Glory program will go through a more traditional integration and test flow following modifications to VCL hardware and procurement of new components. The remaining design and development will be performed in parallel with the rework of the existing components and the SW development.

The bus will also undergo a structural test intended to prove that the existing bus structure can handle the new Launch Vehicle loads. This will be done with the new instrument module assembly (IMA) attached to the SC bus with all the existing components and mass mockups for the instruments, STs and PIP. This testing will include sine and random vibration, as well as separation shock and solar array deployment. This will prove that the existing components can handle the higher Glory loads This testing will also be 
used to derive higher fidelity loads for the instruments since it will occur prior their acceptance testing.

In parallel with the structure fabrication, refurbishment and test, the components will be reworked as required, and tested per ISO procedures. The structure, harness and components will then undergo precision cleaning and a vacuum bake-out in order to ensure that the stringent contamination control and cleanliness requirements are met.

Other efforts will include EDU units of the new builds for the flatsat (EDU's of the various computers and several components in the power subsystem harnessed together to provide a comprehensive flight-like testbed for software and operations script development), simulators of the instruments and bus for interface verifications, as well as a Hybrid Dynamic Simulator (HDS) for ACS algorithm validation. Orbital has stringent configuration control processes that will be implemented during the I\&T process, which includes our problem tracking databases and extensive use of logbooks. In terms of operational robustness, multiple ground stations will be used, as well as the use of the Tracking and Data Relay Satellite System (TDRSS). End-to-End testing will be done with all of the ground system elements. One benefit to the program is the same ground control system is used from the start of integration all the way through the on-orbit operations.

\section{Science Data Processing}

The Glory Project has two science goals, which do not interact scientifically on the spacecraft and really only come together in the global climate models. Aerosol data is collected by the APS Instrument, packetized in CCSDS format, downlinked to the ground and sent bent pipe to the GSFC/GISS Science Data Processing Center (SDPC) for processing and distribution. Data from the $\mathrm{CC}$, once downlinked, is also sent bent piped to the GSFC/GISS SDPC. The GISS SDPC utilizes data from the cloud camera to determine cloud clearing, which is then used for determining global aerosol content. TSI data is collected by the TIM Instrument, also packetized into CCSDS format, donwlinked to the ground station and sent bent pipe to the University of Colorado's Laboratory for Atmospheric and Solar Physics (LASP) Science Operations Center (SOC).

\section{Science Data Collection}

The APS instrument is the primary instrument for collecting aerosol data. The APS SDPC utilizes data from the CC to determine cloud clearing, which is then an input into the aerosol determination equation. The APS is a nadir looking, along-track scanning, optical instrument. Its pixel size is $6.6 \mathrm{~km}$ and it collects data in 9 bands from 412 to $2250 \mathrm{~nm}$. Even though the APS operates continuously, it nominally collects data only during sunlight, when the solar zenith angle is $>70^{\circ}$; no data is retrieved in eclipse. Approximately $490 \mathrm{MBits}$ of data is collected each orbit.
Like the APS, nominal CC data collection operations occur during sunlight when the solar zenith angle is $>70^{\circ}$. Also, like the APS, the CC operates continuously, however no data is retrieved in eclipse. The cloud camera is composed of two cross-track scanning imagers $( \pm 125 \mathrm{~km})$, one band per camera ( $412 \& 865 \mathrm{~nm}$ ). Effective collection time is $44 \%$ of an orbit ( $45 \mathrm{~min})$. Approximately 584Mbits of data (compressed on-board) is collected each orbit.

The TIM instrument collects total solar irradiance data. This instrument utilizes a gimbaled platform to track the sun. Nominal data collection operations occur throughout the entire orbit - TSI data is collected during sunlight while dark (calibration) data is collected during eclipse. Collection cadence is at least once every six hrs. Approximately $18 \mathrm{MBits}$ of data is collected each orbit.

Upon acquisition, all instrument data is formatted into packets as science, housekeeping telemetry, and state of health (SOH). The CCSDS format science data is then stored onboard the spacecraft and at the ground station prior to distribution to the science data processing centers.

Since the Glory Project has two science objectives, two Science Data Processing Centers are utilized to process science data and distribute products to the user community. The GSFC/GISS SDPC processes and distributes aerosol data; therefore, all APS and CC data are sent to this facility from the ground station. The LASP SOC processes and distributes TSI data; as a result, all TSI data collected by the TIM instrument are sent to this facility. Each SDPC is also responsible for archiving science data locally and sending it to the GSFC Data Analysis and Archive Center (DAAC).

The MOC will archive all spacecraft and instrument state of health ( $\mathrm{SOH})$ data. The SDPCs and the MOC are responsible for protecting the data from inadvertent loss until it is archived. The SDPCs and the MOC may also store data for an extended period of time for the convenience of their operations. The MOC will save all archived data for at least five (5) years after the end of the mission. The GSFC/GISS and LASP SDPCs will save archived data and measurements for several years after the end of the mission. GSFC DAAC will be used for long term archiving of mission data and products.

Data may take up to 48 hours to be received for processing from the time of acquisition, and longer latencies are possible if there is an anomaly in the communications. The delay is composed of time it is stored on the spacecraft prior to downlink, time to move the data from the ground station to the SDPC, and time required for level zero processing of the data (remove the packet headers and time order the data).

The length of time the data is stored on the spacecraft is between a few minutes to up to $\sim 28$ hours (one pass per day). The time to get the data from the ground station to the SDPC is nominally 3 hours for the APS and CC, although 
the requirement is $95 \%$ of the data in 24 hours. Approximately $15 \mathrm{Gbits}$ of data per day will be downlinked each day.

Both the GSFC/GISS SDPC and LASP SOC reuse a significant amount of existing algorithms, science and data processing systems (SDPS), and networks for science data processing and distribution. This heritage increases the confidence in the systems and reduces development risk.

The aerosol SDPS leverages existing functionality and utilizes commercial off the shelf products as much as possible. The SDPS is identical to SEAWIFS architecture and utilizes existing codes/tables. Most aerosol specific new algorithms have been tested using aircraft data obtained by the Research Scanning Polarimeter (RSP) Instrument, which has similar characteristics to the APS. The GSFC/GISS SOC will perform some code scrub and performance testing of the aerosol and cloud determination algorithms to assure them for Glory. Cloud mask algorithms, which determine cloud coverage, are simple threshold comparisons. The cloud mask algorithms developed by the RSP will be utilized. Additionally, the CC cloud mask algorithm will use MODIS variability algorithm as currently used in the aerosol algorithm.

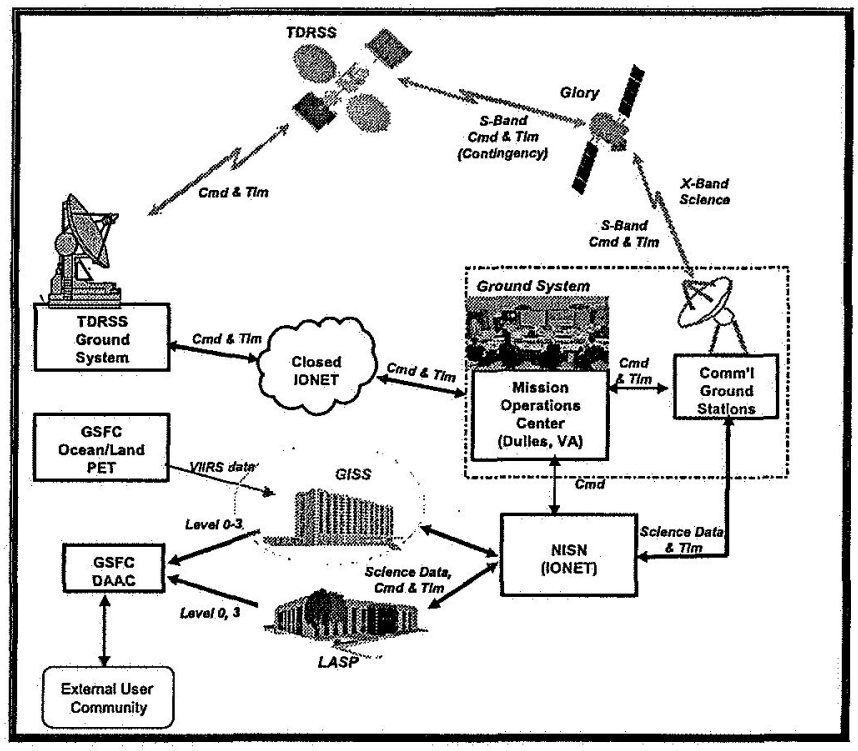

Figure 7 Science Data Processing

The SDPS testing will begin in early 2006, when the APS Instrument vendor will start testing the APS with the spacecraft simulator to verify 1553 compatibility and performance. Once files from this process become available GSFC/GISS will use them to verify format of the APS CCSDS packets and ensure these can be unpacked. The APS Level 0 (L0) to Level 1A (L1A) processing code will be used on the APS calibration and characterization data that is collected to verify that the ground based calibration is consistent with the planned on-orbit calibration of the sensor. The APS L0 to L1A processing code will be used to process the APS data during spacecraft I\&T. Using the processing code on APS data during instrument I\&T will reduce risk of surprises during Observatory level I\&T.

The Glory TIM is primarily a rebuild of the TIM instrument flown on NASA's SORCE mission; the only difference being that the Glory TIM utilizes a pointing platform to continuously track the sun. Therefore, the Glory TIM SDPS is largely a reuse of the one used for the SORCE TIM, which is fully operational and producing a full compliment of TSI data products on a daily basis. Nearly all SORCE and Glory TIM data processing software and algorithm requirements are identical; only a minimal amount of algorithm development is necessary for use of the pointing platform.

TIM software is implemented using an object-oriented design, which isolates algorithms that are independent of one another. This design permits modification/replacement of algorithms with minimal impact on other system elements, thereby reducing long-term maintenance costs. This design was used on SORCE.

\section{Science Data Products}

Each SDPC will process the raw science data, produce data products, and distribute these products to the scientific community. The following data products will be distributed by Glory.

\section{Aerosol Data Products}

- Aerosol optical thickness (fine, coarse, total)

- Aerosol particle size distribution

- Fine mode radius and effective variance

- Coarse mode radius and effective variance

- Aerosol refractive index, single scattering albedo (SSA) and shape

- Fine mode real refractive index and SSA

- Coarse mode real refractive index and SSA

- Non-spherical shape ID and retrieval based on set of prescribed non-spherical models

- Column Water Vapor

- Stratospheric aerosol optical depth and size distribution

- Effective variance and effective radius

\section{Cloud Data Products}

- Liquid cloud optical thickness

- Liquid cloud particle size distribution

- Effective radius and effective variance

- Cloud top height

- Rayleigh barometer used to determine cloud height from 410 and $443 \mathrm{~nm}$ bands.

- Ice cloud optical thickness

- Ice cloud particle size distribution

- Effective radius, effective variance

- Ice cloud shape ID

- ID based on differentiation between set of prescribed mixtures 


\section{Experimental Data Products}

- Water cloud droplet vertical profile ID

- Some information about vertical profile of cloud droplet size is present in data. Depending on how algorithm uses/minimizes this sensitivity some type of profile information will be provided

- Within cloud water vapor amount

- Use $910 \mathrm{~nm}$ polarization measurements to determine how much water vapor absorption is present within the cloud. Subsequent processing will allow cloud vertical extent and cloud droplet number concentrations to be determined.

\section{TSI Data Products}

- Solar irradiance data averaged over 6 and 24 hours at 50 second time cadence.

The scientific community will utilize these products as inputs into the global climate model to meet Glory's scientific goals.

\section{SUMMARY}

The Glory program utilizes government investment through use of assets from a previous mission that was cancelled. In this manner the total program cost for a mission that flies two critical science instruments is minimized. This unique scenario does introduce interesting issues, however, since the state of the bus hardware was not completely known at the start of the program. A rigorous series of tests and audits of all the hardware and paperwork was used to train the new program team and to garner a complete understanding of the state of the hardware. Most of the testing was done at the system level, using the old software to test the component functionality.

The Glory ground data processing system utilizes heritage code and algorithms from existing flight programs to minimize program risk. APS and Cloud Camera data will be processed to Level Zero using Orbital's MAESTRO software, including decompression of the Cloud Camera data. The SeaWIFS data processing system, tailored for Glory, will then be used to further process the data. LASP will process the TIM data using the algorithms and code developed and in use for the SORCE mission. Data will be archived well beyond the end of the mission, as well as on the DAAC.

Glory is a high priority science mission to investigate the causes of global warming through collection of aerosol and irradiance data. As the US contribution to the global Climate Change Research Initiative (CCRI), the APS instrument will investigate both natural and man-made aerosols. The TIM instrument will continue the long-term database of total solar irradiance measurements. The Glory mission will utilize the spacecraft bus originally developed for the VCL mission. Glory will fly on a Taurus launch vehicle from VAFB in late 2008 and operate for at least 3 years.

\section{REFERENCES}

[1] NASA Goddard Space Flight Center, Glory Program Science and Mission Requirements Document, Greenbelt, Maryland, February 2, 2004

[2] D. Durham and T Itchkawich, The Story of Glory: Earth and Solar Science on One Unique Satellite, IEEE Aerospace Conference, March 2005

[3] S. Slanina and Y. Zhang, Aerosols: Connection Between Regional Climatic Change and Air Quality, IUPAC Technical Report, 2004.

[4] NASA Facts, Aerosols, June 1999

\section{BIOGRaphies}

Jaya Bajpayee is the Acting Deputy Project Manager for Glory at NASA's Goddard Space Flight Center in Greenbelt, MD. Her expertise is in design, development,

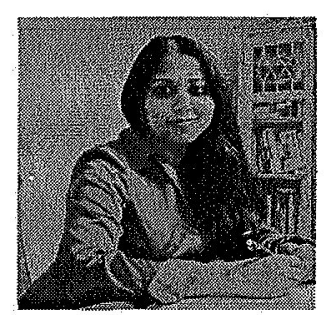

integration, and testing of instruments, spacecraft, and launch vehicles. Her 10 years of experience working launch operations as a Range Safety Officer at NASA's Wallops Flight Facility gave her tremendous experience with flight hardware, launch operations, and making real time destruct/no destruct decisions. She became a nationally recognized expert for Launch Vehicle Flight Termination Systems and served as the Ranige Safety Officer for the first mission which utilized lasers for first stage ignition, second stage ignition, and destruct. In addition to launches in the United States, she supported launch operations at Brazil, Australia, and Spain. As an Instrument Manager for NASA'S GOES Program, she led development of the Solar $X$-Ray Imager Instrument and delivered the first flight instrument within cost, slightly ahead of schedule, and exceeding technical requirements. As the GOES-R Series Observatory Manager she worked extensively with NOAA to define end-to-end requirements for a $\$ 3$ billion mission and led multiagency teams to study a constellation consisting of 8 satellites and 6 different instrument suites.

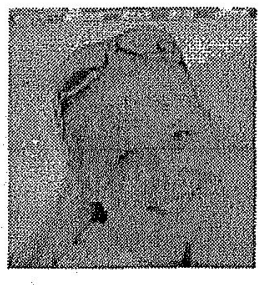

Darcie A. Durham is an Aerospace Engineer in the Advanced Programs Group at Orbital Sciences Corporation in Dulles, Virginia. Currently she is supporting the Glory program in the Systems Engineering Division as well as the Project Lead for a commercial human spaceflight program. Her experience is both on hardware and study programs ranging from ISS Crew 
Preference Items for Lockheed Martin to Crew Exploration Vehicle Design for Orbital Sciences Corporation. She graduated with a B.S. in Aerospace Engineering from Texas $A \& M$ University and is currently pursuing a Masters degree in Applied Physics from George Mason University. Past. experience includes flight certification of ISS hardware at Johnson Space Center in Houston and work with the Advanced Missions Architecture Group at the Jet Propulsion Laboratory in Pasadena, California.

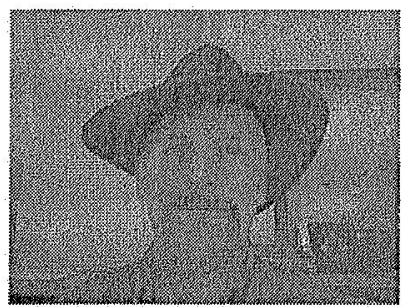

Thomas J. Itchkawich is a Program Director working for the VP of Science and Technology Programs in the Space Systems Group at Orbital Sciences Corporation in Dulles, Virginia. Tom is currently managing the operations support for ACRIMSAT, managing the Glory program at Orbital, and managing an internal ERP conversion in his spare time. He successfully launched the MightySat I satellite on the Shuttle Endeavour and the ACRIMSAT satellite on Taurus. In a previous incarnation he was the lead engineer for the Pegasus payload fairing from inception through the first four flights. A Fighting Blue Hen, Tom graduated with a Bachelor's of Mechanical Engineering from the University of Delaware. The first ten years of his career were spent at Hercules Aerospace working on composite rocket motor cases, including Titan IVB, Delta GEM, and Filament Wound Case Shuttle boosters. Tom was part of the Pegasus Team that was awarded the National Medal of Technology, and the MightySat team that was awarded the Program of the Quarter by AFRL. He has several other published papers on composite structures, low-cost satellite missions and Glory. 